\title{
On Concurrent Transmissions in Multi-hop Wireless Networks with Shadowing Channels
}

\author{
Seung Min Hur ${ }^{\dagger} \quad$ Shiwen Mao ${ }^{\ddagger} \quad$ Kwanghee Nam ${ }^{\dagger}$ Jeffrey H. Reed ${ }^{\S}$ \\ ${ }^{\dagger}$ Div. of Electrical and Computer Engineering, POSTECH, Pohang, 790-784, Republic of Korea \\ ${ }^{\ddagger}$ Dept. of Electrical and Computer Engineering, Auburn University, Auburn, AL 36849, USA

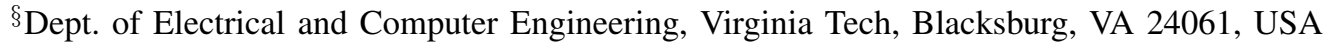 \\ Email: hsm@postech.ac.kr, smao@ieee.org, kwnam@postech.ac.kr, reedjh@vt.edu
}

\begin{abstract}
In this paper, we study the exposed terminal problem in multi-hop wireless networks with log-normal shadowing channels. Assuming that location information is known, we first calculate the success probability for the concurrent transmissions from exposed nodes. We then propose a new MAC protocol which schedules concurrent transmissions in the presence of lognormal shadowing, thus mitigating the exposed terminal problem and increasing network throughput. The performance of the proposed protocol is evaluated with ns-2 simulations, and it is shown to achieve considerable improvements in both end-to-end throughput and delay over the IEEE 802.11 MAC.
\end{abstract}

\section{INTRODUCTION}

It has been shown that IEEE 802.11 MAC suffers low throughput performance in wireless ad-hoc networks [1]. In particular, "exposed terminal problem" deteriorates the network throughput, and it is highly important to solve or alleviate this problem. There has been considerable research effort on this aspect. For example, MAC protocol requiring additional hardware or PHY capacities are shown to be helpful [2]. In addition, there have been proposals on tuning the carrier sensing range [3], controlling the transmit power [4] and modifying the behavior of the IEEE 802.11 MAC [5]. However, these studies are performed with the deterministic wireless propagation models, where path loss is determined by the distance between the transmitter and the receiver deterministically. However, due to obstacles, multi-path propagation, and mobility, randomness exists in most real wireless networks and should be considered in protocol design.

Factors such as reflection, diffraction, and scattering impact the propagation in a wireless network. In addition to power attenuation, "large-scale shadowing" and "small-scale fading" are usually experienced by radio signals. Small-scale fading describes the rapid fluctuation of the signals over a short period of time or distance. On the other hand, large-scale shadowing represents a random effect which occurs over a large number of measurement locations which have the same distance between the transmitter and the receiver, but have different levels of obstacles on the propagation path. It has been known that the log-normal shadowing propagation model captures this characteristic. Some researchers have studied the connectivity problem in presence of log-normal shadowing [6]. To the best of our knowledge, there is no related work considering log-normal shadowing in studying the exposed terminal problem.
In this paper, we study the problem of how to mitigate the exposed terminal problem in the presence of shadowing channels. We first derive an analysis on the success probability of concurrent transmissions from exposed nodes. Here we assume location information for the nodes. Note that such an assumption is reasonable since GPS is becoming more and more accessible. There are also many localization schemes from the literature that can be adopted in case GPS service is not available. We describe a new MAC protocol based on this analysis, aiming to schedule concurrent transmissions in the presence of log-normal shadowing. Such concurrent transmissions can considerably improve the end-to-end throughput and delay performance of multi-hop wireless networks over the IEEE 802.11 MAC, as observed in our ns-2 performance study of the proposed location-assisted MAC protocol.

The rest of this paper is organized as follows. In Section II, we describe the log-normal shadowing propagation model and derive the success probability of a concurrent transmission. We present the proposed MAC protocol in Section III. Our simulation studies are presented in Section IV. Finally, Section V concludes the paper.

\section{Success Probability of a Concurrent TRANSMISSION UNDER SHADOWING}

\section{A. Log-normal Shadowing Propagation Model}

In real wireless networks, the variations in the received power measured at different locations with the same distance from the transmitter, are random and independent [7]. It means that the real transmission range is NOT circular. Fig. 1 illustrates the transmission ranges of the two-ray ground reflection model (left) and the log-normal shadowing propagation model (right), respectively. The variation seen in the right figure is nicely described by the log-normal random variable, and it is incorporated in the log-normal shadowing propagation model.

Consider a pair of transmitter and receiver where the distance between them is $d$. According to the log-normal shadowing propagation model, the received power of the intended signal at the receiver in $\mathrm{dB}, P_{r, d B}$, is represented by

$$
P_{r, d B}=-10 \beta \log \left(\frac{d}{d_{0}}\right)+X_{r}
$$



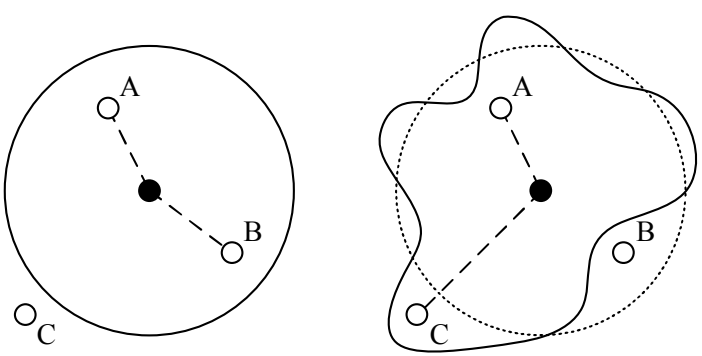

Fig. 1. Transmission ranges: Two-ray ground (left) and shadowing (right).

where $\beta$ represents the path-loss exponent, $d_{0}$ represents the reference distance, and $X_{r}$ represents a normal random variable with zero mean and variance $\sigma_{d B}^{2}\left(=\sigma^{2} /\left(0.01(\ln 10)^{2}\right)\right)$. The received power consists of two parts: the deterministic part depending on the distance between the transmitter and the receiver, and the probabilistic part $X_{r}$. The variation of the received power is determined by the standard deviation $\sigma_{d B}$ or $\sigma$. Note that the standard deviation $\sigma_{d B}$ typically ranges from $4 \mathrm{~dB}$ to $8 \mathrm{~dB}$. The probabilistic part $X_{r}$ makes the transmission range not circular, as shown in Fig 1. The received power can be expressed in unit of watts as follows:

$$
P_{r}=P_{0} 10^{-\beta \log \left(\frac{d}{d_{0}}\right)+0.1 X_{r}}=C \frac{Y_{r}}{d^{\beta}},
$$

where $P_{0}$ is the reference power measured at $d_{0}, C=P_{0} d_{0}^{\beta}$ is a constant, and $Y_{r}=10^{0.1 X_{r}}$ represents a log-normal random variable with zero mean and variance $\sigma^{2}$.

\section{B. Success Probability of the Concurrent Transmission}

Suppose that there are $N$ (active) interfering nodes around a receiver, and that the distance between interfering node $i$ and the receiver is $r_{i}$. Similar to (2), the interference by interfering node $i, P_{i}$, is represented by

$$
P_{i}=C \frac{Y_{i}}{r_{i}^{\beta}}
$$

where $Y_{i}$ is a log-normal random variable with zero mean and variance $\sigma^{2}$. From (3), the cumulative interference $P_{I}$ measured at the receiver amounts to

$$
P_{I}=\sum_{i=1}^{N} P_{i}=\sum_{i=1}^{N} C \frac{Y_{i}}{r_{i}^{\beta}} .
$$

From (2) and (4), we obtain that

$$
\frac{1}{S I R}=\frac{P_{I}}{P_{r}}=\frac{\sum_{i=1}^{N} C \frac{Y_{i}}{r_{i}^{\beta}}}{C \frac{Y_{r}}{d^{\beta}}}=\sum_{i=1}^{N}\left(\frac{d}{r_{i}}\right)^{\beta} \frac{Y_{i}}{Y_{r}} .
$$

In order to receive the packet successfully, the signal-tointerference ratio (SIR) should be greater than a threshold $T_{S I R}$. Thus, we calculate the probability that SIR is greater than a certain threshold $T_{S I R}$.

$$
P_{s}=\operatorname{Pr}\left[S I R>T_{S I R}\right]=\operatorname{Pr}\left[\frac{1}{Y_{r}} \sum_{i=1}^{N}\left(\frac{d}{r_{i}}\right)^{\beta} Y_{i}<\frac{1}{T_{S I R}}\right] \text {. }
$$

Let $U_{i}=\left(\frac{d}{r_{i}}\right)^{\beta} Y_{i}$. Note that $U_{i}$ is a log-normal random variable with mean $\mu_{i}=\ln \left(\frac{d}{r_{i}}\right)^{\beta}$ and variance $\sigma^{2}$. Furthermore, let $V=\sum_{i=1}^{N} U_{i}$. Since $U_{i}$ 's are independent, with the help of Fenton-Wilkinson method [8], $V$ can be approximated by a log-normal random variable $W$ with the following mean $\mu_{w}$ and variance $\sigma_{w}$ :

$$
\left\{\begin{array}{l}
\mu_{w}=\log \left(\sum_{i=1}^{N} e^{\mu_{i}}\right)+\sigma^{2}-\frac{\sigma_{w}^{2}}{2} \\
\sigma_{w}^{2}=\log \left[\left(e^{2 \sigma^{2}}-1\right) \frac{\sum_{i=1}^{N} e^{2 \mu_{i}}}{\left(\sum_{i=1}^{N} e^{\mu_{i}}\right)^{2}}+1\right] .
\end{array}\right.
$$

Finally, $\frac{W}{Y_{r}}$ is a log-normal random variable with mean $\mu_{w}$ and variance $\left(\sigma_{w}^{2}+\sigma^{2}\right)$.

By utilizing the logistic distribution for CDF (cumulative distribution function) of the log-normal distribution [9], $F(x ; \mu, \sigma)=\left[\left(\frac{e^{\mu}}{x}\right)^{\pi /(\sigma \sqrt{3})}+1\right]^{-1}$, we finally obtain that

$$
P_{s}=\left[\left(T_{S I R} e^{\mu_{w}}\right)^{\frac{\pi}{\sqrt{3\left(\sigma_{w}^{2}+\sigma^{2}\right)}}}+1\right]^{-1} .
$$

\section{The Case of a Single Interfering Node}

When an IEEE 802.11-like MAC is used, the area around the transmitter and receiver is reserved by the RTS/CTS handshake. In addition, the carrier sensing range is usually much larger than the transmission range. As a result, the number of interfering nodes around a receiver should be small. Let's consider the case of a single interfering node. Equation (6) reduces to

$$
P_{s}=\operatorname{Pr}\left[\frac{Y_{i}}{Y_{r}}<\frac{1}{T_{S I R}}\left(\frac{r}{d}\right)^{\beta}\right]=\operatorname{Pr}\left[Z<\frac{1}{T_{S I R}}\left(\frac{r}{d}\right)^{\beta}\right]
$$

where $Z$ represents a log-normal random variable with zero mean and variance $2 \sigma^{2}$. Using the logistic distribution, we find the success probability to be

$$
P_{s}=\left[\left(T_{S I R}\left(\frac{d}{r}\right)^{\beta}\right)^{\pi /(\sigma \sqrt{6})}+1\right]^{-1} .
$$

Figure 2 shows the success probability $P_{s}$ for different $\sigma$ 's by evaluating (10). The parameters are selected as $T_{S I R}=10$, $d=20 \mathrm{~m}, r=35.6 \mathrm{~m}$, and $\beta=4$. The mean interference range $R_{I}=d \sqrt[\beta]{T_{S I R}}=35.6 \mathrm{~m}$. We find that $P_{s}$ is a decreasing function of $\sigma$ when $r>R_{I}$, but an increasing function of $\sigma$ when $r<R_{I}$. For example, consider the two extreme cases $\sigma=0$ and $\sigma=\infty$. When $\sigma=0$, (10) tends to be a step function as $P_{s}=\left\{\begin{array}{cc}0, & r<R_{I} \\ 1, & r>R_{I} .\end{array}\right.$ It is the deterministic disk model used in many prior studies. On the other hand, when $\sigma=\infty$, the success probability tends to be $\frac{1}{2}$ in all cases. It means that the success probability is no longer dependent on the distances $d$ and $r$. 


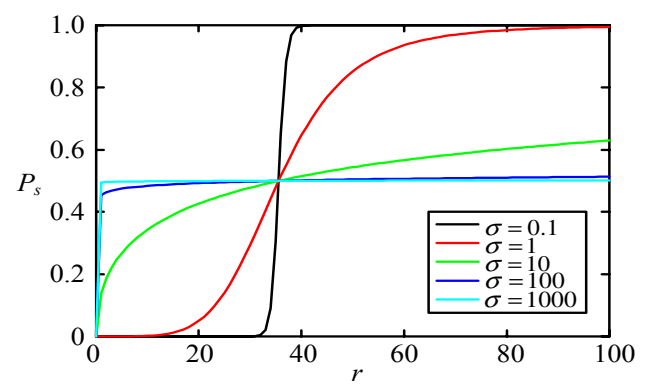

Fig. 2. $\quad P_{s}$ versus $r$ when $T_{S I R}=10, d=20 \mathrm{~m}$, and $\beta=4$.

\begin{tabular}{l}
\hline Procedure validate_schdTx () \\
\hline calculate $P_{D A T A 1}, P_{D A T A 2}, P_{A C K 1}$, and $P_{A C K 2} ;$ \\
if $\left(P_{D A T A 1}>P_{t h} \mathbf{A N D} P_{D A T A 2}>P_{t h} \mathbf{A N D} P_{A C K 1}>P_{t h} \mathbf{A N D} P_{A C K 2}>P_{t h}\right)$ \\
return $1 ; / /$ the scheduled transmission is allowed \\
else \\
$\quad$ return 0; // the scheduled transmission is cancelled \\
endif
\end{tabular}

Fig. 3. Validation procedure.

\section{MAC PROTOCOL FOR CONCURRENT TRANSMISSIONS IN THE PRESENCE OF LOG-NORMAL SHADOWING}

In this section, we describe a MAC protocol which schedules concurrent transmissions under log-normal shadowing channels.

\section{A. Validating a Scheduled Transmission}

Suppose that a node (called "free transmitter") wins the channel and is transmitting to a one-hop neighbor (called "free receiver"). When a node near the free transmitter identifies itself as an exposed terminal, the feasibility of its transmission can be tested probabilistically based on location information.

When testing the feasibility of concurrent transmission, we focus on the case of two (free and scheduled) transmitters and two (free and scheduled) receivers. ${ }^{1}$ Assume that all parameters such as $\beta$ and $\sigma$ are known. ${ }^{2}$ The following four probabilities should be evaluated:

- $P_{D A T A_{1}}$ - the success prob. of the free DATA frame;

- $P_{D A T A_{2}}$ - the success prob. of the scheduled DATA frame;

- $P_{A C K_{1}}$ - the success prob. of the free ACK frame;

- $P_{A C K_{2}}$ - the success prob. of the scheduled ACK frame.

Each probability can be calculated using (10). Fig. 3 shows the validation procedure of the proposed algorithm, where $P_{t h}$ is a prescribed threshold. In order to schedule the concurrent transmission, each of the above probabilities should be greater than $P_{t h}$. As $P_{t h}$ increases, the number of the allowed scheduled transmission decreases.

\footnotetext{
${ }^{1}$ This simplification can be justified by the fact that usually carrier sensing range is much larger than transmission range. The chance of having multiple free transmitters in the neighborhood of the scheduled receiver is small.

${ }^{2} \beta$ and $\sigma_{d B}$ can be estimated like this; (1) can be categorized as the ANOVA model I [10]. According to the least squares criterion, we can obtain the estimators which minimize the error. We omit the detail for the brevity.
}

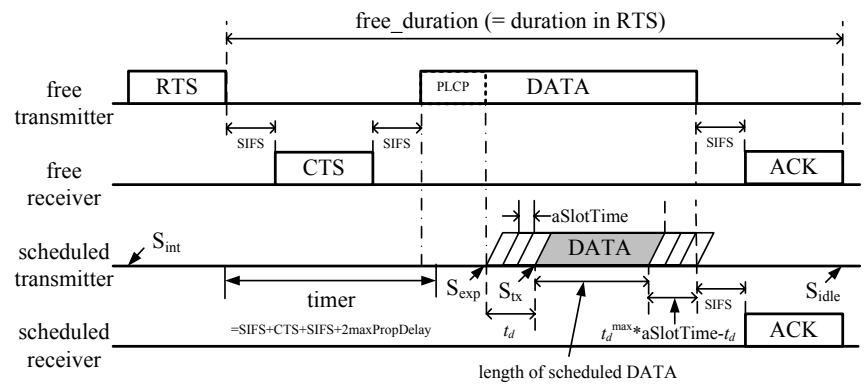

Fig. 4. A time-line illustration of the proposed protocol.

\section{B. The Complete Protocol}

In the proposed scheme, the RTS frame format is modified to piggyback the locations of the sender and its target receiver. When a node overhears an RTS, it extracts the location information, and stores them in a location table.

The operation of the proposed protocol is illustrated in Fig. 4. An exposed node is identified by examining the PHY PLCP frame header, which contains the frame length information, and by double-checking the delay between the RTS and the following DATA frame, which should be $(S I F S+$ $C T S+S I F S)$. Once an exposed node is identified, it will try to validate its concurrent transmission, by executing the validation procedure shown in Fig. 3.

If this test is passed, the exposed node will attempt its scheduled transmission as follows. First, schdTx_margin is calculated as follows:

$$
\begin{aligned}
& \text { schdT } x \_ \text {margin }=\text { free_duration }- \text { SIFS }- \text { CTS }- \\
& \text { SIFS }- \text { PLCP_reading_time }- \text { schd_data_duration - } \\
& \text { SIFS }- \text { ACK }- \text { round-trip_prop_delay }
\end{aligned}
$$

where free_duration is the value in the duration field of the preceding RTS frame. If schdTx_margin is negative, the scheduled transmission will be canceled. That is, the concurrent DATA transmission cannot be aligned with the free DATA transmission. Otherwise, in order to avoid the case of multiple scheduled transmissions in a small neighborhood, a random delay $t_{d}$ is introduced:

$$
t_{d}=\left(\text { random_integer } \% t_{d}^{\max }\right) \times \text { aSlotTime },
$$

where $t_{d}^{\max }=\lceil$ schdTx_margin/aSlotTime $\rceil$.

During the back-off period $t_{d}$, the exposed node will keep on detecting if there is a single transmission (i.e., the free transmission) or multiple transmissions (e.g., a scheduled transmission from another exposed node with a smaller $t_{d}$ value, in addition to the free transmission). If the latter case is detected, the exposed node will not attempt the concurrent transmission; otherwise, it will start sending its DATA frame, which carries the information $T_{\text {info }}$, defined as

$$
T_{\text {info }}=t_{d}^{\max }-t_{d} / \text { aSlotTime. }
$$

When a scheduled transmission is received, the scheduled receiver will return an ACK after a delay of $S I F S+T_{\text {info }} \times$ aSlotTime, in order to align its ACK with the ACK from the free transmission. For more detail, refer to [5]. 


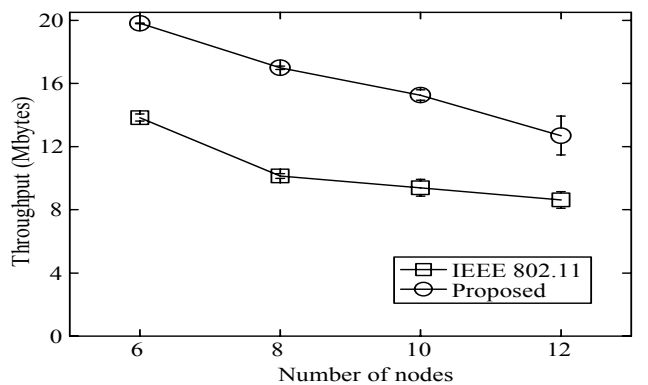

(a) Throughput performance

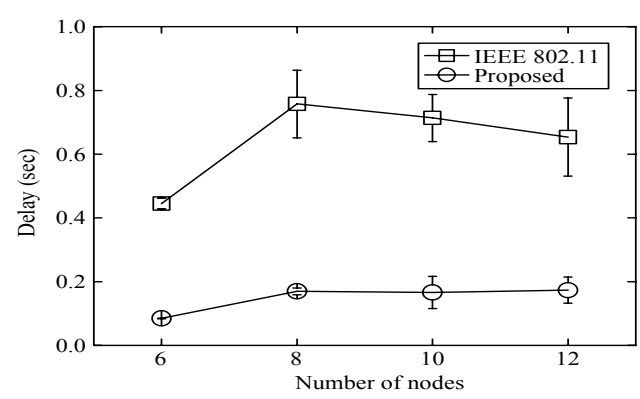

(b) Delay performance

Fig. 5. Simulation results for chain networks when $\sigma_{d B}=0.01$.

\section{Simulation Studies}

We implemented the proposed MAC protocol using the ns -2.30 simulator. Throughout the simulation studies, $P_{t h}$ is fixed to 0.5 . We assume that $\beta=4$ and $\sigma_{d B}=0.01$ or 4 . The ns-2 module "Propagation/Shadowing" is used as propagation model. The channel rate is set to $1 \mathrm{Mb} / \mathrm{s}$. Antenna parameters are set to the default values in $\mathrm{ns}-2.30$. In the simulations, the number of bytes successfully transmitted at the "agent" level (i.e., the end-to-end throughput) and the average end-to-end delay are measured. In each simulation run, all flows start to transmit at $t=10$ second and each simulation lasts for 15 minutes. Each simulation is repeated five times. The averaged results of five trials are presented with $90 \%$ confidence intervals.

\section{A. Chain Networks}

The first simulation study is performed with chain networks. The distance between two adjacent nodes is set to $20 \mathrm{~m}$. The forward flow (from Node 1 to Node $N$ ) is a CBR session with 1000-byte packets, while the backward flow (from Node $N$ to Node 1) is a CBR session with 700-byte packets. The data rates are set to $90 \mathrm{~Kb} / \mathrm{s}, 80 \mathrm{~Kb} / \mathrm{s}, 70 \mathrm{~Kb} / \mathrm{s}$, and $60 \mathrm{~Kb} / \mathrm{s}$ for the 6-, 8-, 10-, and 12-node chain networks, respectively. Note that the mean transmission range is equal to $26.9 \mathrm{~m}$, while the mean carrier sensing range is equal to $59.3 \mathrm{~m}$. When the distance between the transmitter and the receiver is equal to $20 \mathrm{~m}$, the mean interference range is equal to $35.6 \mathrm{~m}$.

Consider first the case that $\sigma_{d B}=0.01$. In this case, the received power is almost the same as the mean value. Thus, a transmitter hardly reach the nodes except for their one-hop neighbors. For example, when $d=20$ and $r=40$, the success

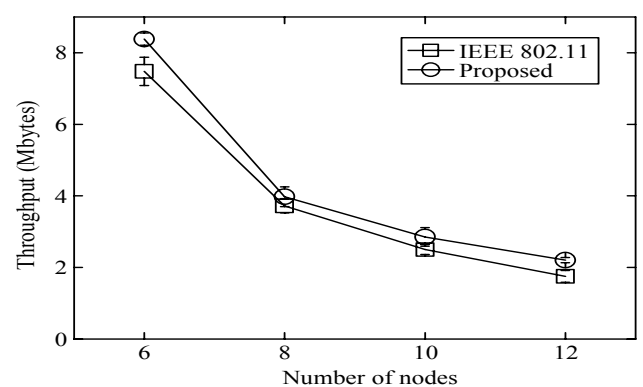

(a) Throughput performance

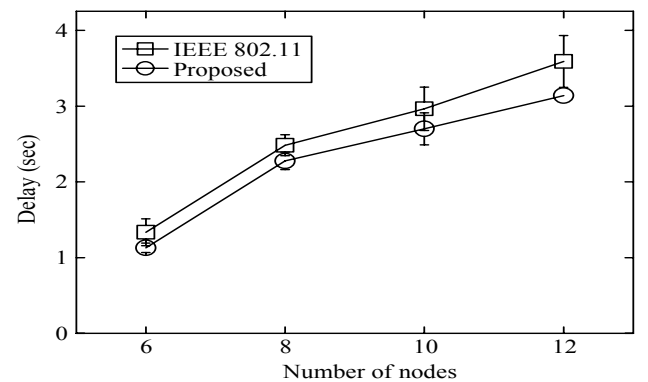

(b) Delay performance

Fig. 6. Simulation results for chain networks when $\sigma_{d B}=4$.

probability of the concurrent transmission is $P_{i} \approx 1$, where $i$ can be $D A T A_{1}, D A T A_{2}, A C K_{1}$, or $A C K_{2}$.

Figure 5 shows the simulation results. We observe considerable throughput improvements achieved by the proposed protocol. Specifically, $43.2 \%, 67.7 \%, 62.4 \%$, and $47.3 \%$ normalized throughput improvements are achieved for the 6-, 8-, 10-, and 12-node chain networks, respectively. In addition, the average end-to-end delay is also drastically reduced for all the cases. From Fig. 5(b), we find that the ratios of the average delay of the proposed MAC to that of the IEEE 802.11 MAC are $19.1 \%, 22.4 \%, 23.3 \%$, and $26.5 \%$, respectively.

We next consider the case of $\sigma_{d B}=4$. Since the average distance between the transmitter and the receiver gets longer, the distance between the receiver and the interfering node should also be longer to prevent the collision. Even if the distance between the transmitter and the receiver is small, the success probability decreases due to large $\sigma_{d B}$. For example, when $d=20$ and $r=40$, the success probability is $P_{i}=$ 0.5376 , where $i$ can be $D A T A_{1}, D A T A_{2}, A C K_{1}$, or $A C K_{2}$. It is smaller than that when $\sigma_{d B}=0.01$. Consequently, there are fewer feasible concurrent transmissions when $\sigma_{d B}$ is large.

Figure 6 shows the results for $\sigma_{d B}=4$, where the normalized throughput improvements are $12.0 \%, 6.9 \%, 14.1 \%$, and $25.7 \%$, respectively. From Fig. 6(b), we find the ratios of the average end-to-end delays are $84.7 \%, 91.7 \%, 91.1 \%$, and $87.5 \%$, respectively. Compared to the case of $\sigma_{d B}=0.01$, the number of scheduled transmissions are reduced. However, the proposed MAC protocol still achieves considerable improvements in end-to-end throughput and delay. 


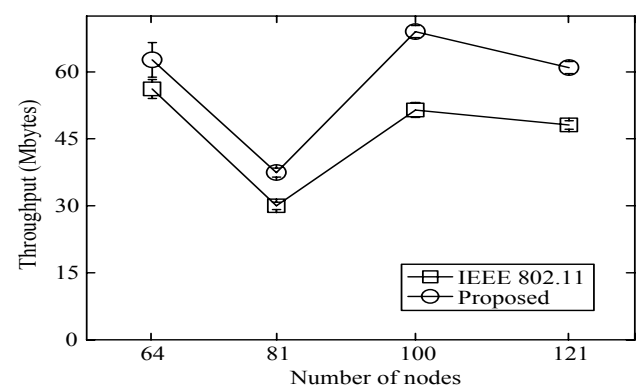

(a) Throughput performance



(b) Delay performance

Fig. 7. Simulation results for random networks when $\sigma_{d B}=0.01$.

\section{B. Random Networks}

We also study the proposed MAC protocol using networks with random topologies. Node locations and traffic flows are randomly generated as follows: $2 n$ nodes are selected as the sources for $n^{2}$-node random network. A half of sources transmit a CBR traffic of 1000-byte packets, while the rest of sources transmit that of 700-byte packets.

Simulation results for the case of $\sigma_{d B}=0.01$ are plotted in Fig. 7. From Fig. 7(a), we find that normalized throughput improvements of $11.6 \%, 24.8 \%, 34.1 \%$, and $26.7 \%$ are achieved. In addition, we find from Fig. 7(b) that the ratios of the average end-to-end delay with the proposed MAC to that of the IEEE 802.11 MAC are 91.8\%, 87.2\%, 81.4\%, and $88.8 \%$, respectively. The simulation results for the case of $\sigma_{d B}=4$ are presented in Fig. 8. We find that normalized throughput improvements of $25.3 \%, 34.1 \%, 42.2 \%$, and $51.2 \%$ are achieved. We also find from Fig. 8(b) that the ratios of the average end-to-end delay with the proposed MAC to that of the IEEE $802.11 \mathrm{MAC}$ are $81.1 \%, 81.8 \%, 78.8 \%$, and $70.3 \%$, respectively. The proposed MAC protocol achieves considerable improvements in both throughput and delay for the random networks.

\section{CONCLUSION}

In this paper, we study the problem of improving the throughput of multi-hop wireless networks with log-normal shadowing channels. We first derived the success probability for the concurrent transmissions from exposed terminals, and then proposed a location-assisted MAC protocol, which validates the feasibility of the concurrent transmission based on the calculated success probability and a prescribed threshold value. Our ns-2 simulation results showed that the proposed

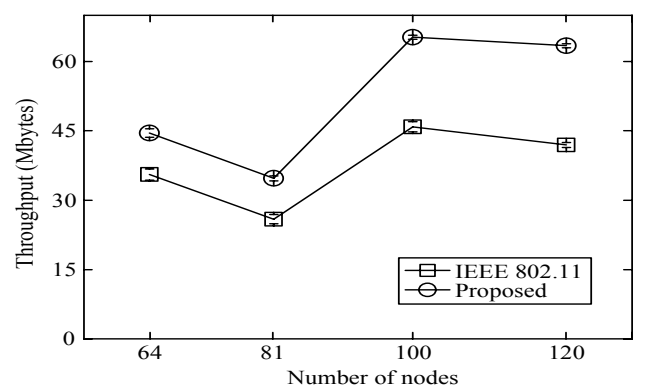

(a) Throughput performance

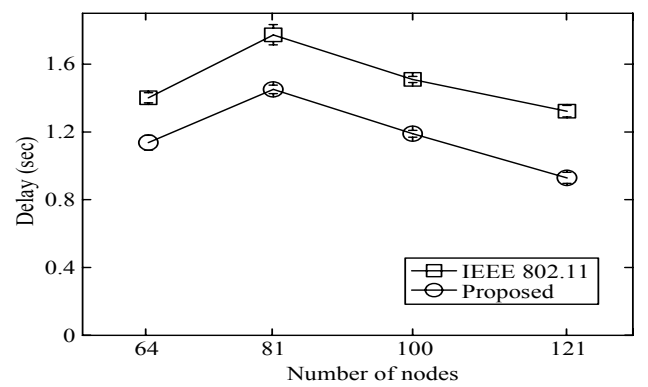

(b) Delay performance

Fig. 8. Simulation results for random networks when $\sigma_{d B}=4$.

protocol can effectively leverage spatial reuse, and thus improve the throughput and delay performance for IEEE 802.11 based multi-hop wireless networks.

\section{ACKNOWLEDGMENTS}

This work was supported in part by the National Science Foundation Wireless Internet Center for Advanced Technology.

\section{REFERENCES}

[1] J. Li, C. Blake, D. S. J. D. Couto, H. I. Lee, and R. Morris, "Capacity of ad hoc wireless networks," in Proc. ACM MobiCom'01, 2001, pp. 61-69.

[2] J.-S. Park, A. Nandan, M. Gerla, and H. Lee, "SPACE-MAC: Enabling spatial reuse using MIMO channel-aware MAC," in Proc IEEE International Conference on Communications (ICC), May 2005, pp. 36423646.

[3] H. Zhai and Y. Fang, "Physical carrier sensing and spatial reuse in multirate and multihop wireless ad hoc networks," in Proc. IEEE INFOCOM '06, Apr. 2006.

[4] Y. Zhou and S. M. Nettles, "Balancing the hidden and exposed node problems with power control in CSMA/CA-based wireless networks," in Proc. IEEE Wireless Communications and Networking Conference (WCNC), vol. 2, Mar. 2005, pp. 683-688.

[5] S. M. Hur, S. Mao, Y. T. Hou, K. Nam, and J. H. Reed, "On Exploiting Location Information for Concurrent Transmissions in Multihop Wireless Networks," accepted to IEEE Trans. Veh. Technol., 2008.

[6] R. Hekmat and P. V. Mieghem, "Connectivity in wireless ad-hoc networks with a log-normal radio model," Mobile networks and applications, vol. 11, pp. 351-360, June 2006.

[7] D. Kotz, C. Newport, R. S. Gray, J. Liu, Y. Yuan, and C. Elliott, "Experimental evaluation of wireless simulation assumptions," in Proc. MSWiM, 2004, pp. 78-82.

[8] L. F. Fenton, "The sum of log-normal probability distributions in scatter transmission systems," IEEE Trans. Commun., vol. 8, pp. 57-67, Mar. 1960.

[9] P. K. Swamee, "Near lognormal distribution," Journal of Hydrologic Engineering, vol. 6, pp. 441-444, 2002.

[10] J. Neter, M. H. Kutner, C. J. Nachtsheim, and W. Wasserman, Applied Linear Statistical Models, 4th ed. WCB/McGraw-Hill, 1996. 